\title{
Análisis estructural de contenidos: Herramientas básicas para su comprensión y uso
}

\author{
María Angélica Rodríguez LLona*
}

\begin{abstract}
RESUMEN
El presente trabajo muestra, de manera didáctica, un método de análisis cualitativo: el Análisis Estructural de Contenido, que a diferencia de los métodos tradicionales es, a su vez, un método y una teoría sociológica, ya que al mismo tiempo que propone un procedimiento de trabajo, se dirige a la construcción de un determinado "objeto", que permitirá describir y construir los principios que organizan los modelos culturales y que tienen como referencia las representaciones a través de las cuales los actores definen su medio, se construyen a sí mismos y actúan.
\end{abstract}

Palabras clave: Análisis cualitativo / lenguaje del MAE / procedimiento a seguir / estructuras del MAE y sus propiedades.

\section{Structural analysis of contents: basic tools for their understanding and use}

\section{ABSTRACT}

The present work describes, in a didactic fashion, a method for qualitative analysis: the Structural Analysis of Contents that, unlike the traditional methods, is both a method and a sociological theory since, while it proposes some working procedures, it aims at the construction of a pre-established "target", which will allow us to describe and to construct the principles that organize the cultural models that have, as a reference framework, the representations, through which the actors define their environment, they construct themselves, and finally, they act out.

Key words: Qualitative Analysis / MAE language / follow-up procedures / MAE structures and properties

* Chilena. Trabajadora social, Magister en Sociología. Docente escuela de Trabajo Social Universidad Católica Silva Henríquez. Correo electrónico: mrodrig@ucsh.cl 


\section{Introducción}

El presente trabajo pretende mostrar, de manera didáctica, un método de análisis cualitativo: el Análisis Estructural de Contenido. Sin embargo, a diferencia de los métodos tradicionales, es posible afirmar que el análisis estructural es, a su vez un método y una teoría sociológica, ya que al mismo tiempo en que propone un procedimiento de trabajo, se dirige a la construcción de un determinado "objeto", que nos permitirá describir y construir los principios que organizan los modelos culturales y que tienen como referencia las representaciones a través de las cuales los actores definen su medio, se construyen a sí mismos y actúan.

Para todo profesional de las Ciencias Sociales resulta un reto enfrentar metodológicamente las expresiones de los puntos de vista de los actores sociales en un campo de acción determinado, cuestión inseparable de la contextualización cultural de éstos, en el entendido de enfocar la cultura como un conjunto estructurado de ideas, de valores, que orientan subjetivamente las conductas y determinan la aproximación que un grupo tiene de la realidad o de su realidad, la forma en que él define los problemas y su solución.

Lo que resulta de interés para el analista serán los principios de ordenamiento del mundo y lecturas de sí mismo, que no son propios de un individuo, sino que revelan lógicas culturales mucho más generales, por cuanto lo particular de la cultura es su capacidad de producir distinciones simbólicas de la realidad, las que variarán según los diferentes grupos sociales en un momento histórico determinado. Será obvio señalar que, en todo dominio de la vida social y en todas las etapas del desarrollo de la humanidad, los actores han desplegado y despliegan formas de conocer y de interpretar la realidad, lo que configura un determinado modelo cultural, es decir, todo actor participa y contribuye a la conformación de un modelo simbólico determinado y dicho modelo será una manifestación de sentidos culturales codificados que a su vez influirá en las conductas de dichos actores.

Es posible situar el Método de Análisis Estructural' ${ }^{1}$, desarrollado por Jean Pierre Hiernaux, Doctor en Sociología de la Universidad Católica de Lovaina (Bélgica), dentro de los intentos de elaboraciones teóricas y metodológicas de dicha problemática, aunque a diferencia de

1 Utilizaremos la sigla M.A.E. para referirnos al Método de Análisis Estructural. 
los tradicionales métodos de análisis cualitativo de contenidos, su aporte fundamental es la pretensión no sólo de comprender los contenidos subyacentes en un determinado soporte (discurso, entrevista, imagen, etc.), sino también la búsqueda de la estructura simbólica de su producción, intentando como metodología, extraer el sentido o el sistema de percepciones inmerso en un soporte determinado.

\section{Algunos conceptos fundamentales}

Como se ha mencionado anteriormente, el propósito del MAE será la extracción del sentido que se encuentra en un soporte o discurso determinado, para lo cual se comenzará por definir -primeramentelos conceptos básicos con que se trabajará, es decir, en qué consisten las unidades de sentido, su totalidad y su estructura, según el método presentado.

El MAE parte del principio que ningún elemento encuentra su significación en sí mismo, suponiendo la puesta en relación de al menos dos elementos. Así, este método reposa sobre un postulado de naturaleza binaria, según el cual todo contenido puede ser reconstruido a través de una "caja de herramientas", cuyo instrumento mínimo es una relación de dos términos. Esta relación fundamental lleva el nombre de relación de disyunción o código disyuntivo.

A modo de ejemplificación, supongamos que en un material dado encontramos el elemento "occidente". Para construir el código disyuntivo debemos encontrar el elemento que se opone a "occidente" (es decir, que entra con él en una relación de disyunción). Este elemento puede ser el "oriente". Ahora bien, la relación entre estos elementos no se agota en su mera disyunción o relación de oposición. Implica también una relación de suposición mutua (para concebir el occidente hay que concebir también el oriente. El occidente sólo tendrá sentido en su oposición al oriente). En otras palabras, tenemos una relación complementaria de presuposición mutua.

Es por ello que el MAE postula que un término² se constituye en

2 Usamos la palabra "término", pues no hemos encontrado una más apropiada; sin embargo, reconocemos que la motivación lexical que nos aporta puede manifestar "efectos perversos", en el entendido de comprenderse como "un solo término". Sin embargo lo que nosotros hemos denotado como "término" puede ser un conjunto de palabras, imágenes, etcétera, es decir, que las unidades buscadas son independientes de las unidades lingüísticas en el caso que trabajemos con textos. Una unidad 
la disyunción u oposición con otro término, el cual -a su vez- será su inverso, a condición de que ambos pertenezcan a una totalidad común, la cual J. P. Hiernaux define como el punto de vista desde el cual se organiza la oposición de los términos en relación. En nuestro ejemplo, podríamos considerar la disyunción occidente/oriente como los extremos opuestos de un intervalo que expresará la "posición geográfica", siendo esta la totalidad común que les aporta un sentido.

$\begin{array}{lll}\text { Definimos entonces: } & \mathrm{A}=\text { Occidente } \\ & \mathrm{B}=\text { Oriente } \\ & =\text { Relación de disyunción u oposición } \\ & \mathrm{A}+\mathrm{B}=\text { Totalidad }\end{array}$

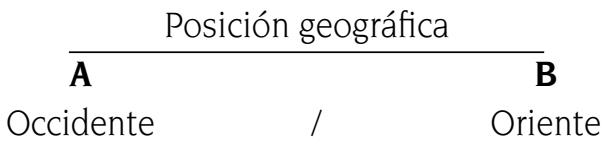

El MAE definirá, como unidad básica del sentido, el código disyuntivo, el que consistirá en la definición de dos términos (donde al menos uno de ellos se encuentra en el soporte), los cuales se encuentran en una relación de oposición y de presuposición mutua, es decir, serán las partes dicotómicas de una totalidad. Resulta interesente señalar que un término puede encontrarse al inicio de un material y su opuesto puede aparecer al final, ya que, para el MAE, el orden aparente en el material no es importante y no es necesario dividirlo en secuencias de análisis; diferencia importante de este método con respecto a otros más tradicionales.

Sin embargo, es posible suponer que si encontramos en el material un elemento o término A, su inverso pudiera no existir en el soporte. Si el inverso de A no se identifica directamente o no aparece en el material, diremos entonces que A se opone a $\sim$ A, lo que nos permitirá avanzar una hipótesis sobre la identidad del inverso, la cual, como toda hipótesis, podrá ser refutada o confirmada durante nuestro análisis.

Para el MAE, un elemento sólo o aislado no tiene significación, ya

puede manifestarse en un material textual bajo formas diversas, como secuencias de palabras, verbos, sustantivos, adjetivos, etcétera. Vale lo mismo para otros soportes o materiales. 
que quien constituirá el código disyuntivo es precisamente esta relación, que expresará el sentido más elemental expresado en el soporte. Esto implica, como hemos dicho anteriormente, que el principio fundamental de construcción del MAE es de naturaleza binaria.

Cuando se trabaje con materiales voluminosos es preciso buscar técnicas que simplifiquen la identificación de las unidades de sentido, ya que pueden ser expresadas a través de diferentes términos. Es por ello que se debe proceder a operaciones de condensación para hacer pasar a un término único lo expresado por una pluralidad de términos. Recordemos, no obstante, la importancia del respeto por parte de quien investiga a lo expresado en el material y la necesidad de no aventurar hipótesis de manera inmediata que podrían no corresponder con el sentido expresado. Se recomienda buscar mucha información y tratar de obtener la definición de los elementos y de sus contrarios cuidadosamente, y sólo en casos extremos, aventurar hipótesis.

Para continuar nuestro razonamiento, es importante señalar que las expresiones humanas son mucho más complejas en la realidad que lo que puede mostrar un simple tratamiento de las oposiciones entre sus términos, y es por ello que el MAE plantea, en segundo lugar, la propiedad que tienen los códigos disyuntivos de encontrarse caracterizados por operaciones de asociación entre sus unidades elementales.

Es necesario destacar sin embargo, que en términos teóricos, la disyunción u oposición es considerada primaria, anterior a la asociación, porque las unidades de sentido van a nacer de sus oposiciones y después es que se asocian, es decir, es imposible la real comprensión de una noción si obviamos su contrario.

Supongamos un material en forma de texto donde hemos detectado dos códigos disyuntivos: mujer/hombre y suavidad/rudeza. Supongamos, además, que la suavidad se encuentra atribuida como una característica de la mujer y la rudeza como una característica del hombre. El MAE diría entonces que se produce una asociación entre mujer-suavidad y otra entre hombre-rudeza. Las asociaciones serán tantas como características o propiedades sean expresadas en el material. El tratamiento de las asociaciones pasará por las mismas atenciones que el de las disyunciones. 
Trabajar de esta manera nos lleva a plantearnos una nueva interrogante. Nótese que los códigos disyuntivos señalados en el ejemplo anterior no son de la misma naturaleza. Aquí, el MAE hará una nueva distinción entre los códigos disyuntivos objeto (mujer, hombre) y los códigos disyuntivos de calificación (suavidad, rudeza). Los códigos objeto, como se puede apreciar, expresarían las partes dicotómicas de una totalidad que a continuación va a ser descrita, y los calificativos serán las características propias a los códigos objeto. Vale señalar, sin embargo, que los códigos disyuntivos de calificación serán también las partes dicotómicas de una totalidad "calificativa". Podemos comprender entonces que, entre los códigos objeto y los códigos de calificación, se establecerán relaciones de asociación.

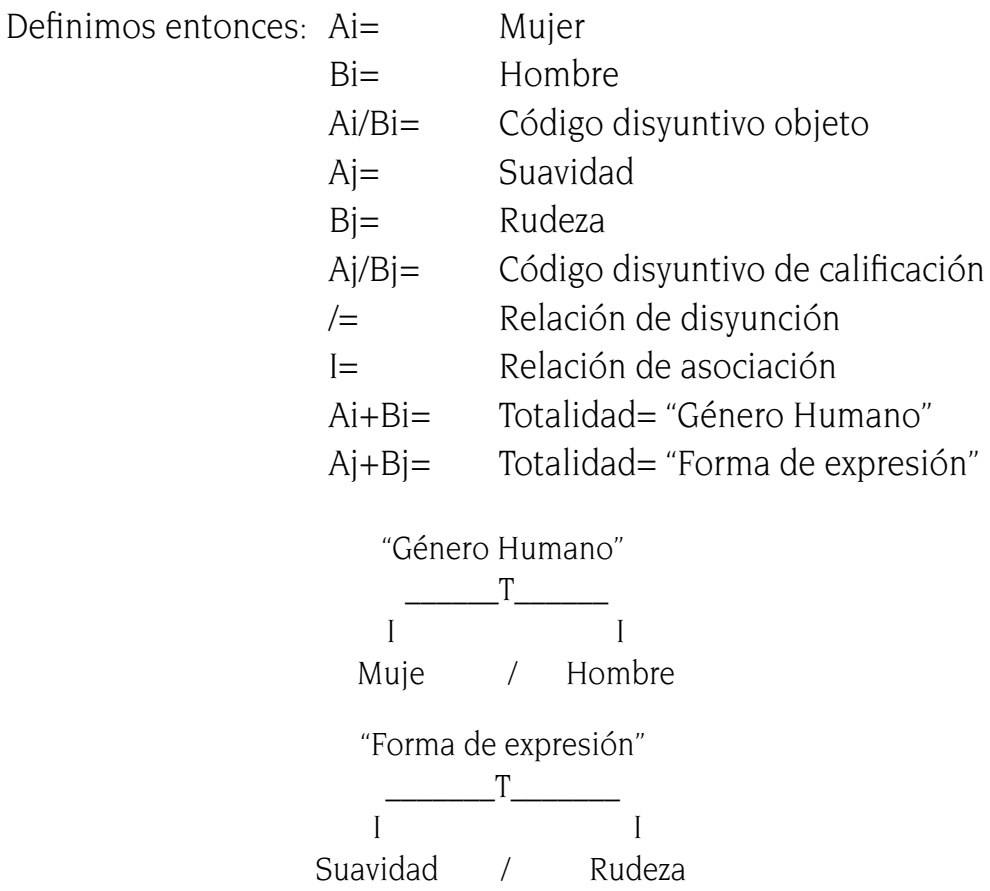

Considerando que nos encontramos en una perspectiva estructuralista, pasaremos a un nivel superior de abstracción del MAE, el cual tratará de derivar de un soporte una o varias estructuras, es decir, un conjunto estructurado de elementos que no tienen sentido por sí solos, pero que cobrarán significación en su relación con otros.

Una estructura, concepto clave en el MAE, será una combinación de elementos que exige, al menos, la existencia de dos códigos disyun- 
tivos en relación. La estructura podrá ser definida como un ordenamiento lógico entre los códigos disyuntivos que permita establecer y comprender las relaciones entre ellos. El sentido de un término será igual a su posición en la estructura y éste sólo existe en las circunstancias del material concreto, es decir, el sentido expresado en el material se mostrará a través del sistema de relaciones que conforman las unidades de sentido, e inversamente, dichas unidades de sentido sólo pueden concebirse en la estructura.

El análisis del contenido de un soporte, según el MAE, se realizará en dos fases: la primera, será la construcción y descripción de las unidades elementales del sentido y, la segunda, el establecimiento de las relaciones entre ellas y su dinámica. La noción de isotopía, definida como el lugar común donde viven un conjunto de códigos, donde diferentes términos se encuentran reunidos bajo una cierta coherencia o un cierto punto de vista, será entonces clave para una cabal comprensión de este método. Será evidente, por tanto, que no se puedan describir estructuras con códigos disyuntivos que no pertenecen a la misma isotopía.

\section{El lenguaje del MAE}

Con el objeto de introducir al lector en la metodología utilizada por el MAE, se ofrecerá a continuación un conjunto de precisiones.

Denotaremos, en el MAE, un código disyuntivo por los términos A y B. Ello implica que A sería el inverso de B, y B sería el inverso de A. Como trabajaremos con múltiples códigos disyuntivos, diremos entonces $\mathrm{Ai} \mathrm{Bi}$, donde i podrá variar de 1 hasta $\mathrm{n}$.

La disyunción se expresará con el símbolo / y la asociación con el símbolo I. La totalidad con la letra $\mathrm{T}$ y los diferentes planos calificativos (aquellas que contienen los códigos disyuntivos de calificación) se denominarán qi $(i=1, n)$. El plano que contiene los códigos disyuntivos objeto se denominará q0 y puede ser situado al inicio o al final de la estructura.

La condensación de determinados términos en uno de nuestro propio lenguaje exigirá que el nuevo término deba ser colocado entre comillas, lo que indicará que no forma parte del vocabulario utilizado en el material. Cuando aventuremos una hipótesis, ésta debe ser colocada entre paréntesis. 


\section{Resumiendo:}

\begin{tabular}{|l|l|}
\hline Ai/Bi: & Códigos disyuntivos, Ai se opone a Bi. \\
\hline $\mathrm{A}=\sim \mathrm{B}$ y B= A: & A es igual al inverso de B y B es igual al inverso de A. \\
\hline Ai-Aj: & Ai se asocia a $\mathrm{Aj}$ \\
\hline T=A+B. & $\begin{array}{l}\text { T es la totalidad común que expresa el sentido de A y B. El sig- } \\
\text { no + debe interpretarse en el sentido de la unión matemática. }\end{array}$ \\
\hline q0 : & $\begin{array}{l}\text { Instancia o plano donde se sitúan los códigos disyuntivos ob- } \\
\text { jeto. }\end{array}$ \\
\hline qi : & $\begin{array}{l}\text { Instancia o plano donde se sitúan los códigos disyuntivos de } \\
\text { calificación. }\end{array}$ \\
\hline "C": & $\begin{array}{l}\text { C es un término condensado que no se encuentra en el mate- } \\
\text { rial. }\end{array}$ \\
\hline$(\mathrm{H}):$ & Hes una hipótesis que aventuramos. \\
\hline
\end{tabular}

Finalizaremos con una última precisión: si en el material fuera imposible encontrar el inverso de A (caso del inverso vacío), éste será definido como $\sim \mathrm{A}$; por el contrario, si quien apareciera en el texto fuese $\sim$ A, podríamos definir inmediatamente su inverso como A.

\section{Algunos elementos de procedimiento a seguir en el MAE}

El método de análisis estructural debe cumplir ciertas normas y principios de procedimiento. Los momentos operatorios específicos son los siguientes:

Construcción del gráfico:_Un gráfico es la transposición a una modalidad gráfica de la estructura de códigos que puede extraerse de un determinado material, explicitando de esta manera las relaciones que allí se manifiestan. Será una forma de visualización del trabajo de codificación efectuado por el investigador y se hará de acuerdo al lenguaje y reglas definidas por el MAE.

Paráfrasis: La paráfrasis es un test destinado a comprobar si la descripción que hemos realizado se ajusta o no a los contenidos del material. Parafrasear consiste entonces en "leer" el gráfico para verificar si la repetición oral que hacemos de su estructura se ajusta o no al contenido del soporte. Esto debe hacerse respetando lo que expresa el material, a pesar de que pueda entrar en contradicción con nuestro propio sistema de sentidos.

Protocolo analítico:_Equivale al momento intelectual que consiste 
en traducir el gráfico en una lengua natural (ejemplo: el español), es decir, expresando exactamente cómo se estructuran los códigos en el gráfico. Puede ser concebido también como una formulación -en un lenguaje accesible- de los núcleos de sentido puestos de manifiesto y relacionados por la estructura del gráfico.

Comentarios analíticos: Este momento será el de la reflexión sociológica y consistirá en extraer todas las consecuencias que trae consigo la estructura de sentido puesta de manifiesto por los procedimientos anteriores. Ello nos mostrará las consecuencias implícitas que subyacen en la estructura de sentido y que escapan a la percepción corriente.

Los principios básicos que debe respetar la descripción estructural son la conformidad y la canonicidad.

- La conformidad es el respeto al contenido concreto del material en cada selección de unidades de sentido que realicemos, así como en cuanto a las asociaciones que describamos. La forma de descripción no debe alterar el sentido contenido en el material, sino que debe transmitirlo con la mayor fidelidad posible.

- La canonicidad, por su parte, se refiere al respeto de las reglas de descripción definidas, es decir, la axiomática del método, cuyos aspectos centrales fueron tocados en el punto anterior.

El respeto de estos principios obedece a la necesidad de potenciar procedimientos de descripción y análisis estandarizados que faciliten la comunicación entre investigadores y potencien -a la vez- la replicabilidad de investigaciones cuya perspectiva teórica y metodológica es similar.

\section{Las estructuras del MAE y sus propiedades}

Las estructuras que se expondrán a continuación podemos entenderlas como órdenes o configuraciones típicas, a través de las cuales pueden aparecer organizados los sistemas de sentido. El MAE propone cinco tipos de estructuras, con propiedades comunes en algunos casos y particulares en otros. Esta parte de nuestro trabajo tratará de mostrarlas de la manera más comprensible al lector.

La Estructura Cruzada: Esta será la estructura "típica" del MAE y todas las demás serán sus casos particulares con algunas propieda- 
des que establecen relativas diferencias a señalar. En efecto, la estructura cruzada ofrece todas las posibilidades teóricas que surgen de una combinación dada de códigos disyuntivos. En una palabra, dicho tipo de estructura topologiza y construye un espacio de sentido dentro del cual las otras estructuras no representan otra cosa que configuraciones particulares.

Podemos afirmar, entonces, que nos encontramos frente a una estructura cruzada, cuando existen al menos dos códigos disyuntivos, de manera tal que cada uno de los términos del primer código disyuntivo se asocia simultáneamente a los dos términos del segundo código disyuntivo.

\section{a) Su forma gráfica ${ }^{3}$}

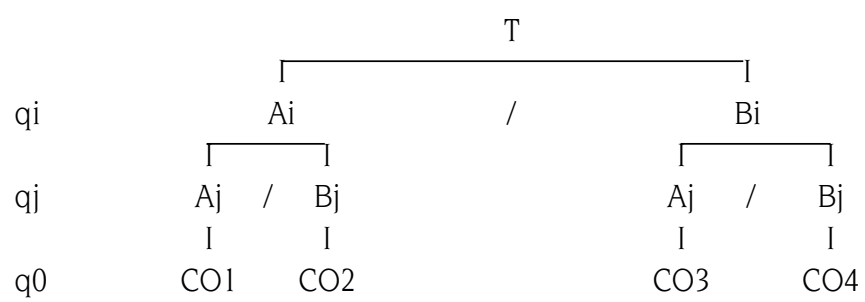

Sin embargo, el orden de ocurrencia de los códigos disyuntivos de calificación no interviene en el problema del sentido que expresa la estructura cruzada, lo que nos induce a mostrar la siguiente estructura que sería tan válida como la anterior para la misma pareja de códigos disyuntivos Ai/Bi Aj/Bj

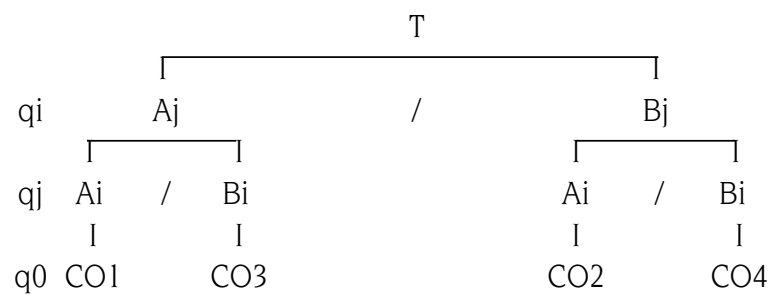

Una forma sencilla de visualizar lo que es una estructura cruzada es imaginar dos códigos disyuntivos y pensar que, al cruzarlos, ellos

3 Vale aclarar que en el resto del trabajo nos limitaremos a mostrar las estructuras en la segunda dimensión para facilitar la comprensión del método, lo que no quiere decir que en el trabajo cotidiano tengamos que reducirnos a $n=2$. 
concurren en la formación de un espacio bidimensional, como lo muestra el siguiente gráfico:

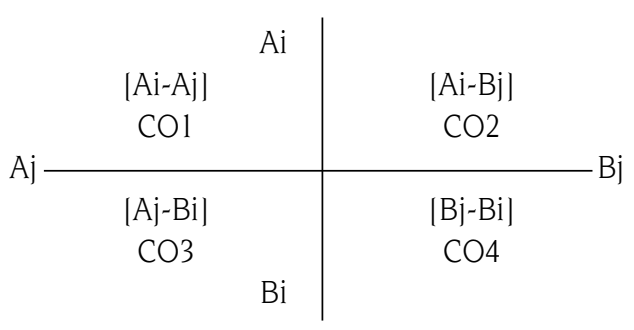

\section{b) Sus propiedades}

1) Para que exista una estructura cruzada debe existir al menos dos códigos disyuntivos de calificación que den lugar a cuatro objetos. Es necesario que $n>=2$, es decir, que existan al menos dos planos.

2) Si Ai se asocia a $\mathrm{Aj} / \mathrm{Bj}$ entonces, Bi se asocia a $\mathrm{Aj} / \mathrm{Bj}$.

3) $\mathrm{Si} A=\sim \mathrm{B}$ entonces $\mathrm{B}=\sim \mathrm{A}$

4) $A+B=T$, en el sentido de la unión matemática.

5) Si no aparece el inverso de A; asumimos que sería A y podríamos establecer la hipótesis que $\sim A$ sería igual a $B$, es decir, A / $\sim \mathrm{A}_{\text {; }}(\mathrm{B})$.

6) Si quien aparece en el texto es $\sim$ A y su inverso es vacio, entonces podremos decir que su opuesto es A.

7) El orden de inscripción de los códigos disyuntivos no modificará el contenido de los términos. Esto implica que una lectura del gráfico que suponga una secuencia cronológica, sería un error.

\section{Un ejemplo}

Para clarificar la estructura cruzada, proponemos utilizar como ejemplo la teoría sobre las lógicas de la reciprocidad social desarrollada por Guy Bajoit, profesor de la Universidad Católica de Lovaina, Bélgica.

"Cuando los actores G (gestionario) son dirigentes (cuando ellos se ocupan eficazmente del bien colectivo) las finalidades que ellos persiguen son aceptadas por los actores P (populares), que entran con ellos en intercambios inclusivos: ninguno puede alcanzar su finalidad sin la colaboración del otro.

Al contrario, cuando los actores $G$ son dominantes (cuando ellos se ocupan de sus finalidades privadas) ellos imponen a los actores $\mathrm{P}$ intercambios exclusivos: solamente pueden alcanzar su finalidad impidiéndole al otro alcanzar la suya. 
Lo que se aplica a los beneficiarios de las desigualdades se emplea también con aquellos que las sufren. En su resistencia, los actores P son, a la vez, ofensivos $y$ defensivos.

Son defensivos cuando ellos reconocen el orden desigual establecido como bien fundado, es decir, cuando las desigualdades son consensuales.

En el caso contrario, cuando ellos son ofensivos (cuestionan las desigualdades), entonces las desigualdades son disensionales y los actores son adversarios. Esto da lugar a cuatro intercambios diferentes entre los actores P y G: complementario, conflictual, competitivo y contradictorio.

A continuación expondremos los cuatro tipos de intercambio que se establecen entre los actores P y G, a través de la siguiente estructura cruzada:

ql

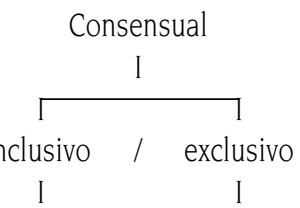

q0 Complementario Competitivo

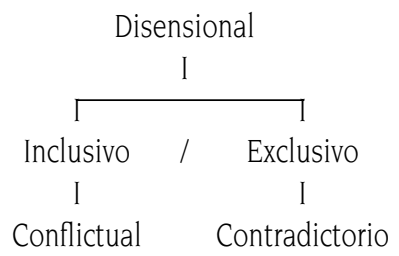

Donde,

q1: Tipo de desigualdad que se establece entre los actores P y G.

q2: Finalidad de los intercambios entre los actores P y G.

q0: Instancia objeto. Tipo de intercambio entre los actores P y G.

En un sistema cartesiano, podríamos graficar esta misma estructura, como se muestra en el esquema siguiente:

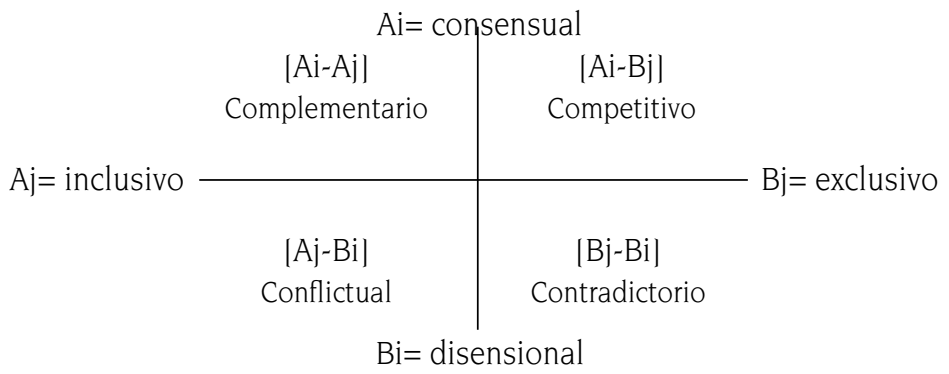




\section{La Estructura Paralela}

Será el tipo más sencillo de estructura que podemos encontrar en el MAE y un caso particular de la estructura cruzada. Puede ser definida como la estructura donde existen al menos dos códigos disyuntivos, de manera tal que, si el primer término del primer código disyuntivo se asocia al primer término del segundo código disyuntivo, implica que los dos restantes se asocian entre ellos. Estamos en presencia de una estructura paralela toda vez que un material presenta un contenido comparativo mutuamente excluyente o de tipo dicotómico. En una estructura paralela, la asociación de todos los términos clasificados a la izquierda del gráfico y de todos los términos clasificados a la derecha del mismo gráfico, definen un "mundo", es decir, un universo que engloba esos términos. Todo lo que es dicho respecto a un Universo A se contradice en relación a un Universo B. Estos dos universos se contradefinen mutuamente.

\section{Su gráfico:}

\begin{tabular}{|c|c|c|c|}
\hline & & $\begin{array}{c}\mathrm{T} 1 \\
\mathrm{I}\end{array}$ & \\
\hline & I & & \\
\hline qi & $\mathrm{Ai}$ & I & $\mathrm{Bi}$ \\
\hline & I & & I \\
\hline 4) & $\mathrm{Aj}$ & 1 & $\mathrm{Bj}$ \\
\hline & I & & I \\
\hline & $\mathrm{CO}$ & & $\mathrm{CO} 2$ \\
\hline
\end{tabular}

Podremos imaginarnos la transposición de esta estructura a un plano cartesiano:

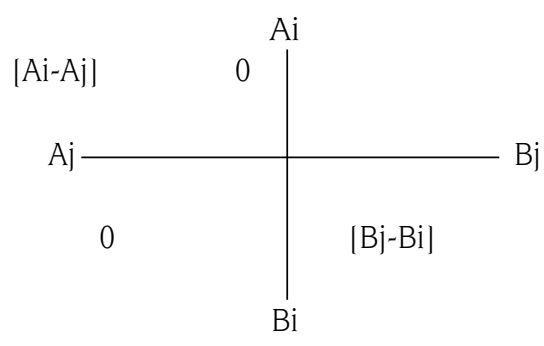

Sus propiedades:

1) Para que exista una estructura paralela debe existir -al menosun código disyuntivo objeto y uno de calificación. Es necesario que $n>=2$, es decir, que existan al menos dos planos. 
2) $\mathrm{Si}$ Ai se asocia a $\mathrm{Aj}$, entonces $\mathrm{Bi}$ se asocia a $\mathrm{Bj}$.

3) $\mathrm{Si} A=\sim B$ entonces $B=\sim A$

4) $A+B=T$, en el sentido de la unión matemática.

5) Si no aparece el inverso de $A$; asumimos que sería A y podríamos establecer la hipótesis que $\sim$ A sería igual a $B$, o sea, $A / \sim A$; (B).

6) Si quien aparece en el texto es $\sim$ A y su inverso es vacio, entonces podremos decir que su opuesto es A.

7) El orden de inscripción de los códigos disyuntivos no modificará el contenido de los términos. Esto implica que una lectura del gráfico que suponga una secuencia cronológica, sería un error.

\section{Un ejemplo:}

Ilustramos la estructura paralela, a través de un proverbio bíblico (Proverbios 6 del 6-11), titulado: El perezoso y la hormiga:

Vete donde la hormiga, perezoso,

mira sus andanzas y te harás sabio.

Ella no tiene jefe,

ni capataz, ni amo:

asegura en el verano su sustento,

recoge su comida al tiempo de la mies.

¿Hasta cuándo, perezoso, estarás acostado?

¿Cuándo te levantarás de tus sueños?

Un poco dormir, otro dormitar,

otro poco tumbarse con los brazos cruzados:

y llegará como vagabundo tu miseria

y como un mendigo tu pobreza.

Del proverbio citado, podemos derivar la siguiente estructura paralela:

$\begin{array}{cccc}\text { q1 } & \text { Asegura su sustento } & / & \sim \text { Asegura su sustento } \\ \text { q2 } & \text { Recoge su comida } & , & \sim \text { Recoge su comida } \\ \text { q3 } & \sim \text { Acostada, } \sim \text { duerme } \\ \text { (Trabaja) } & / & \begin{array}{c}\text { Acostado, duerme } \\ \text { (no trabaja) }\end{array} \\ \text { q4 } & \begin{array}{c}\sim \text { Miseria, } \sim \text { pobreza } \\ \text { (riqueza) }\end{array} & , & \text { Miseria, pobreza } \\ & \text { qormiga } & , & \text { Perezoso }\end{array}$


Si observamos la estructura de códigos que pudimos distinguir analíticamente en este ejercicio, veremos que varios de los códigos de calificación no presentaban en el material la presencia de ambos términos de la disyunción. Ello ocurre con bastante frecuencia en los materiales concretos y de allí la necesidad, para el investigador, de determinar adecuadamente el término contradefinido con una realidad que está presente en el material, ya sea a nivel de códigos de objeto o de códigos de calificación. En el caso del ejemplo presentado, frente a la realidad: Acostado, duerme, dormita, nos hemos visto en la necesidad de levantar una hipótesis respecto a la realidad contradefinida que concurre a la constitución del código. Así, hemos notado: (trabaja), indicando con el paréntesis el carácter hipotético de ese elemento extraído indirectamente del material.

Asimismo, las características asociadas a la hormiga y al perezoso nos parecen redundantes, por lo que proponemos una visualización bidimensional que daría lugar a la siguiente estructura paralela y su representación espacial cartesiana de la siguiente manera:

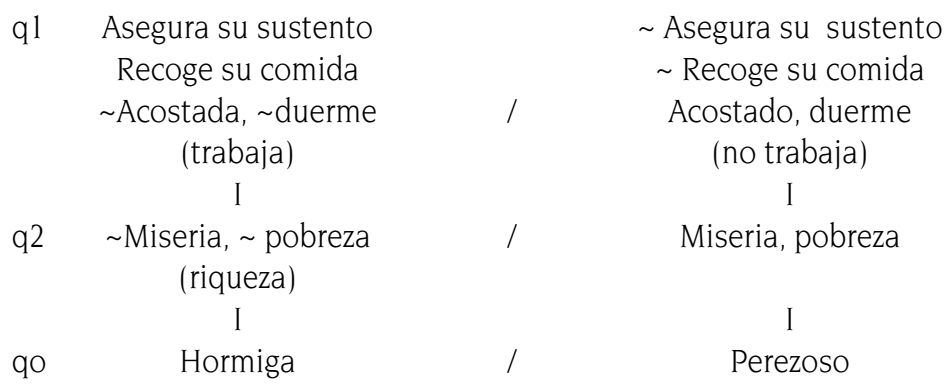

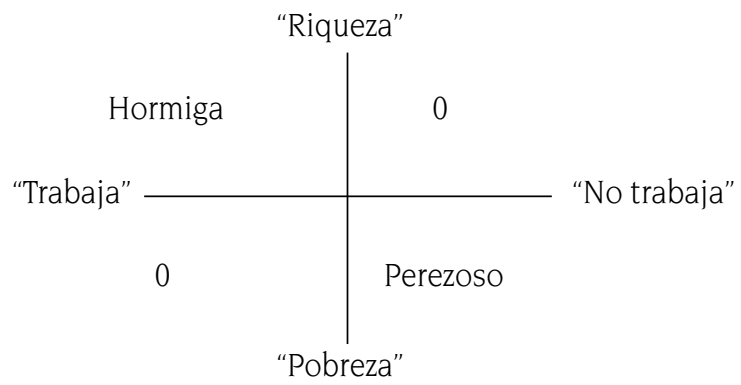

Este ejemplo nos permite apreciar con claridad el problema del sentido. Obsérvese que en el proverbio bíblico se expresa con claridad la valoración positiva del trabajo como fuente de riquezas. Si planteamos nuestra representación cartesiana ajustando a los ejes un 
signo más (+) si la valoración es positiva y un signo menos (-) si es negativa, veremos que los objetos que se conforman van a llevar en sí mismos una connotación significante:

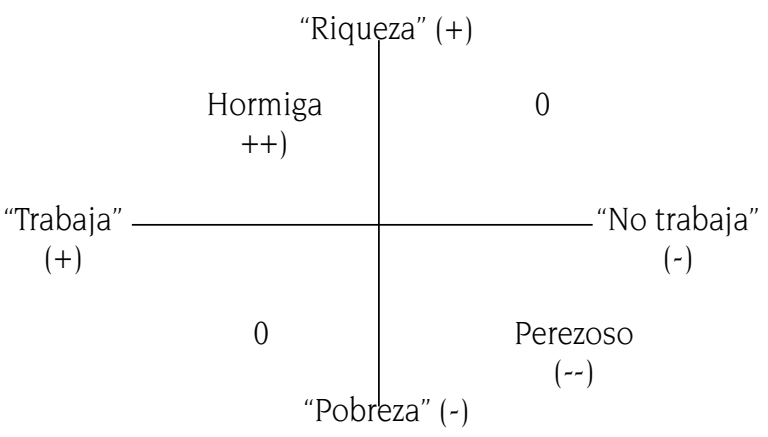

\section{La Estructura en abanico}

La estructura en abanico puede considerarse también como un caso particular de estructura cruzada y será aquella donde existen al menos dos códigos disyuntivos, de manera tal que, si Ai se asocia a $\mathrm{Aj} / \mathrm{Bj}$, entonces Bi no se asocia a ninguno. Si la estructura cruzada nos situaba en el dominio del entrecruzamiento de propiedades, la estructura en abanico, en cambio, nos impone la secuencialidad de las disyunciones.

Su gráfico:

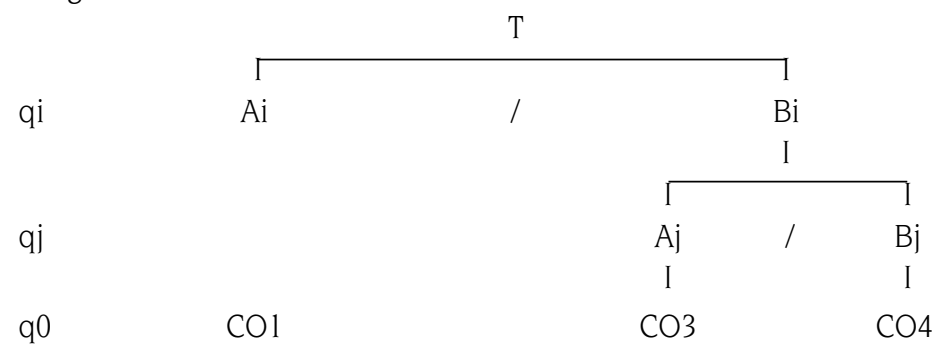

En el plano cartesiano:

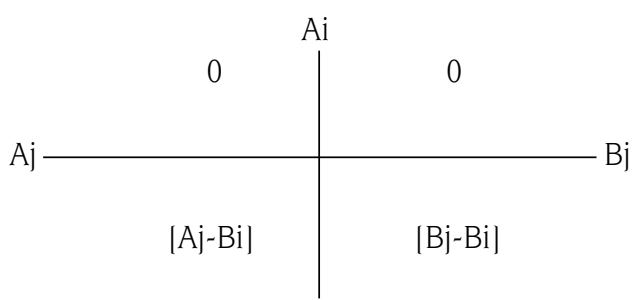

$\mathrm{Bi}$ 


\section{Sus propiedades:}

1) Para que exista una estructura en abanico debe existir al menos dos códigos disyuntivos de calificación que den lugar a tres objetos. Es necesario que $n>=2$, es decir, que existan al menos dos planos.

2) Si Ai se asocia a $\mathrm{Aj} / \mathrm{Bj}$, entonces $B i$ no se asocia a ninguno.

3) $\mathrm{Si} \mathrm{A}=\sim \mathrm{B}$ entonces $\mathrm{B}=\sim \mathrm{A}$

4) $A+B=T$, en el sentido de la unión matemática.

5) Si no aparece el inverso de $A$; asumimos que sería $\sim A$ y podríamos establecer la hipótesis que $\sim$ A sería igual a B, o sea, A / A; (B).

6) Si quien aparece en el texto es $\sim A$ y su inverso es vacio, entonces podremos decir que su opuesto es A.

7) El modo constitutivo en abanico implica una secuencialidad lógica, un orden de intervención. La Estructura en abanico es una reducción de las posibilidades teóricas ofrecidas por la estructura cruzada, introduciendo en los códigos una secuencialidad que excluye o reduce ciertos componentes en beneficio del encajamiento jerarquizado de aquellos que son retenidos.

8) La estructura en abanico muestra 3 códigos objeto donde un tercero no será consecuencia del entrecruzamiento de [Ai-Aj] ó [Ai-Bj], sino que el hecho de ocurrir Ai ya define un código objeto. En este caso, la representación cartesiana no contribuirá a esclarecer el problema del sentido, sucediendo lo contrario con el gráfico.

\section{Un ejemplo:}

Intentaremos ejemplificar esta estructura analizando una situación o construcción "objetivada", lo que contribuirá a mostrarnos las potencialidades del MAE. Recordemos que antes de la caída del Muro de Berlín, el mundo podía dividirse fundamentalmente en tres bloques: los países que pertenecían a la OTAN o potencias occidentales, los países del Pacto de Varsovia o campo socialista y los países No Alineados. La pertenencia o no a una organización militar internacional y su tipo nos permite caracterizar la correlación de fuerzas a nivel mundial que se observaba después de la segunda guerra mundial hasta el derrumbe del campo socialista. Veamos como podemos representar esta situación a través de una estructura en abanico: 

q1
p pertenecen
/ pertenecen

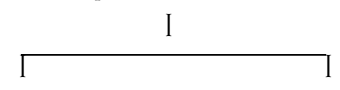
q2
OTAN
Pacto de Varsovia
q0 No Alineados
I
I
P. Occidentales
Campo Socialista

donde:

q1: Pertenencia a una organización militar internacional

q2: Tipo de organización

q0: Categoría de países

Obsérvese, a través del siguiente ejemplo, la secuencialidad o lectura cronológica que exige la estructura en abanico:
q1 OTAN
Pacto de Varsovia
q2

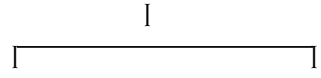
q0 P. Occidentales

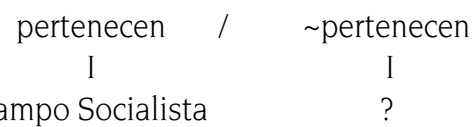

En el plano cartesiano su representación sería la siguiente:

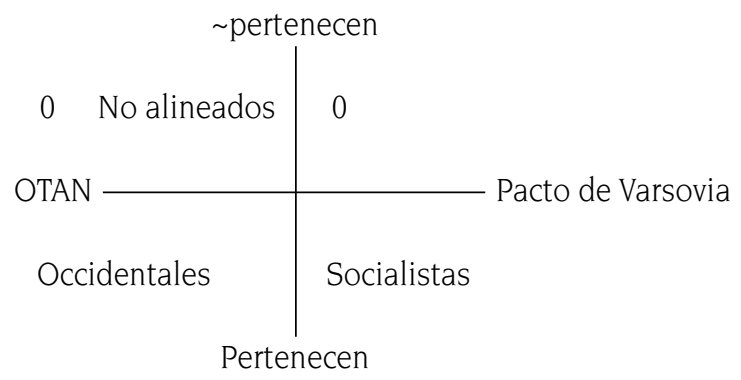

\section{La Estructura Truncada:}

La estructura truncada puede considerarse como otro caso particular de estructura cruzada que podemos encontrar en el MAE. Podemos definirla como aquella donde existen al menos dos códigos disyuntivos (Ai/Bi Aj/Bj), de manera tal que si Ai se asocia a Aj/Bj entonces Bi solamente se asocia a uno de los dos términos del código disyuntivo $\mathrm{Aj} / \mathrm{Bj}$. 


\section{Su gráfico:}

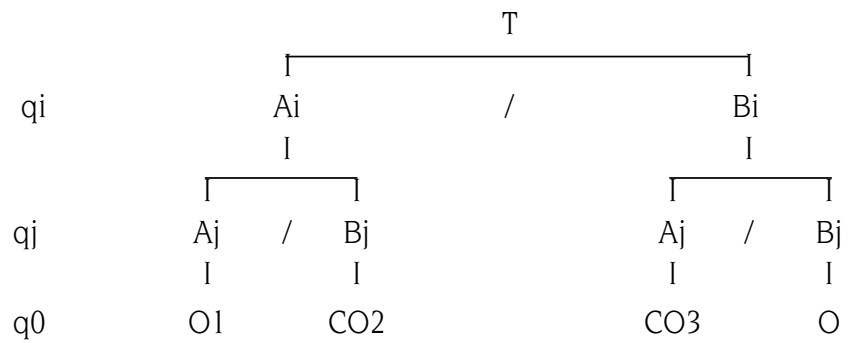

Y en el dominio cartesiano:

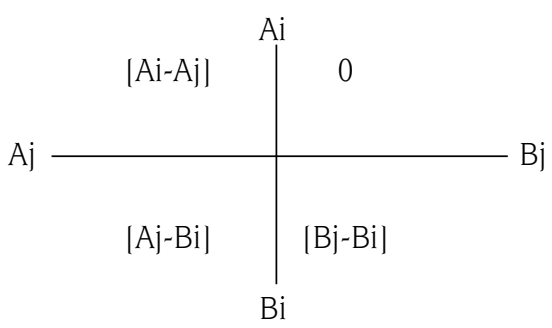

\section{Sus propiedades:}

1) Para que exista una estructura truncada debe existir al menos dos códigos disyuntivos de calificación que den lugar a tres objetos. Es necesario que $n>=2$, es decir, que existan al menos dos planos.

2) Si Ai se asocia a Aj/Bj entonces Bi solamente se asocia a uno de los dos términos del código disyuntivo Aj/Bj, es decir, se asocia o a $\mathrm{Aj}$ o a $\mathrm{Bj}$.

3) $\mathrm{Si} A=\sim \mathrm{B}$ entonces $\mathrm{B}=\sim \mathrm{A}$

4) $A+B=T$, en el sentido de la unión matemática.

5) Si no aparece el inverso de $A$; asumimos que sería $\sim A$ y podríamos establecer la hipótesis que $\sim$ A sería igual a $B$, o sea, A / A; (B).

6) Si quien aparece en el texto es $\sim A$ y su inverso es vacio, entonces podremos decir que su opuesto es A.

7) El orden de inscripción de los códigos disyuntivos no modificará el contenido de los términos. Esto implica que una lectura del gráfico que suponga una secuencia cronológica, sería un error, a diferencia de la estructura en abanico.

\section{Un ejemplo:}

En la vida universitaria Belga y específicamente en el Departamento de Sociología de la UCL hay tres categorías fundamentales respec- 
to a los títulos otorgados: la Licencia en Sociología, los Diplomas (complementario y "avanzados") y el Doctorado en Sociología. Según el tipo de enseñanza: grado o postgrado y según su reconocimiento en el "mercado laboral universitario", podemos representar esta clasificación según la siguiente estructura truncada:

ql

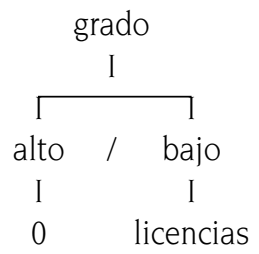

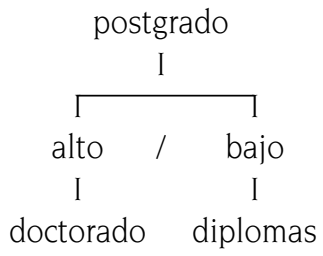

Donde:

q1: Tipo de diploma que oferta la UCL

q2: Grado de valoración del diploma en el mercado laboral universitario

q0: Diploma ofrecido

En el dominio cartesiano la representación será:

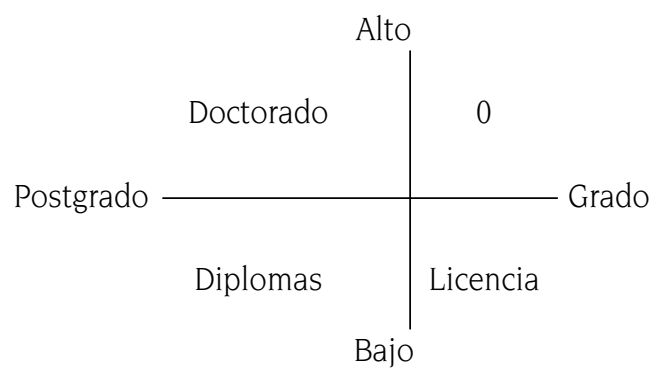

\section{El esquema actancial:}

Las estructuras vistas anteriormente podemos clasificarlas como instrumentos descriptivos, ya que sólo nos brindan información a nivel cognitivo. Pasaremos ahora a explicar una nueva estructura: el esquema actancial, el cual se inbricará con determinadas problemáticas teóricas que explicaremos más adelante.

El esquema actancial surge a partir de la problemática expuesta por A. J. Greimas con respecto a determinadas regularidades encontradas en múltiples análisis. El Profesor J. P. Hiernaux, introdujo aportes que permitirán presentar el esquema actancial en la versión que 
mostraremos a continuación. Su nombre se debe a que la representación gráfica es mostrada a través de actantes, es decir, de posiciones definidas según las funciones que cumplen las oposiciones.

El esquema actancial resulta un tipo específico de estructura paralela, o mejor dicho, una manera de representar la dinámica que se establece en determinadas estructuras paralelas. Ellas deben contener un código disyuntivo de calificación que muestre un sujeto, en sus potencialidades positivas, que se encuentra en la búsqueda de un objeto en sus cualidades positivas y obviamente que rechace al sujeto y al objeto considerados negativos. El código disyuntivo objeto se operacionaliza a través de acciones concretas, las cuales, si son positivas, conllevarán al sujeto al encuentro de su objeto positivo y viceversa. Otra instancia de calificación mostrará que existen "cosas" susceptibles de ayudar al sujeto a cumplir sus acciones positivas y, por el contrario, que existen también "cosas" que se oponen a esto. Otra instancia, también calificativa, permitirá conocer que existen entidades que pueden aparecer a los ojos del sujeto como quienes lo reenvían a las acciones positivas o a las negativas.

\section{Su gráfico:}

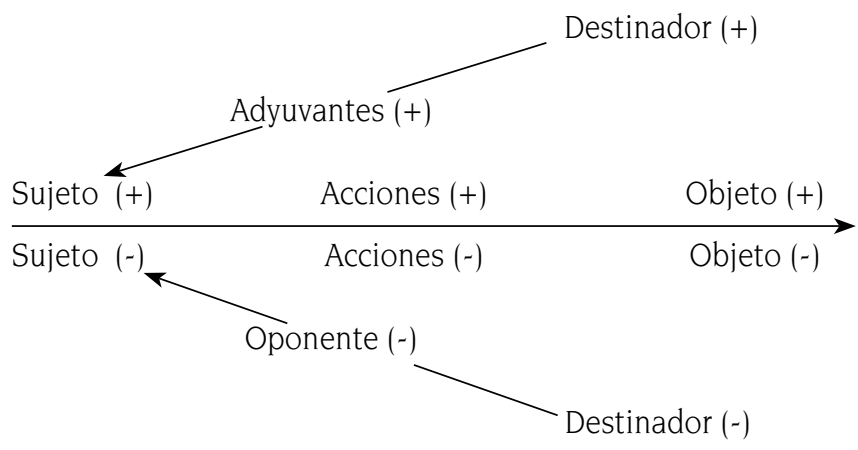

\section{Algunas reflexiones sobre el esquema actancial}

La estructura paralela será releída a otro nivel según este esquema. La función psico-afectiva se definirá en relación a los roles establecidos en términos de acción.

- El sujeto que busca un objeto se califica por una falta, por una carencia en sí mismo. Cuando se está en la búsqueda de un objeto se expresa una carencia y, más allá, esto muestra una cierta invalidación, una falta de capacidades del sujeto. El sujeto que 
efectúa la búsqueda es -en sí mismo- aquel que se encuentra en posición negativa.

- Los adyuvantes y oponentes serán las proyecciones de la impaciencia de acción del sujeto. El adyuvante será quien compensará la falta de potencialidades iniciales. El sujeto le dará entonces al adyuvante un poder en su capacidad de hacer, que tendrá como origen su propia incapacidad de hacer.

- Detrás del objeto buscado hay, al mismo tiempo, la realización, la instauración del sujeto en sus potencialidades positivas.

- En los destinadores habrá una proyección de la detención de poder, la que se traducirá en el poder negativo -en el caso del destinador negativo-, lo que no quiere decir que sea la ausencia de poder. Los destinadores serán los detentores del poder, mientras que los adyuvantes/oponentes serán el poder en sí mismos.

Es en estas proposiciones que podemos ver cómo la producción del sentido puede explicarse a través de la dinámica psico-afectiva, la articulación existente entre la economía psíquica, a partir de la producción de sentido. La dimensión psico-afectiva y el sentido también pueden comprenderse a partir de lo social. Todos los elementos convocados en el esquema actancial son elementos o nociones que forman parte de la realidad social, de la cultura imperante. Ellos son realidades sociales en tanto construcciones, representaciones establecidas culturalmente. Si nuestro esquema se ajusta a las lógicas culturales, son las realidades sociales las que se expresan en él, en el sentido de que nos ofrece la articulación de las dinámicas psico-afectivas y el orden social o modelo cultural imperante. Es evidente que los objetos de búsqueda del sujeto surjan a partir de las condiciones sociales y culturales que lo entornan.

\section{Un ejemplo:}

Hace algunos años, durante un curso de Religión, Cultura y Sociedad, brindado por el propio Profesor J. P. Hiernaux, nos dimos a la tarea de analizar un discurso que hiciera el ex Presidente Salvador Allende, durante el período de la Unidad Popular, en Chile. La estructura paralela que muestra la simbólica cultural expresada en sus discursos puede resumirse de la manera siguiente: 


\begin{tabular}{|l|l|c|l|}
\hline & \multicolumn{1}{|c|}{-} & & \multicolumn{1}{|c|}{+} \\
\hline Sujeto & "Hombre Capitalista" & $/$ & "Hombre Socialista" \\
\hline Acciones & Acciones de dominación & $/$ & Acciones liberadoras \\
\hline Medios & $\begin{array}{l}\text { "Vía de la fuerza y } \\
\text { violencia" }\end{array}$ & $/$ & $\begin{array}{l}\text { "Vía política, democrática, } \\
\text { vía chilena" }\end{array}$ \\
\hline Alimentadores & $\begin{array}{l}\text { Clase dominante } \\
\text { aliada al "Imperialismo" }\end{array}$ & $/$ & $\begin{array}{l}\text { Pueblo, representado en la } \\
\text { Unidad popular }\end{array}$ \\
\hline Objeto & $\begin{array}{l}\text { "Opresión social” } \\
\text { Capitalismo }\end{array}$ & $/$ & Vida=Liberación Socialismo \\
\hline
\end{tabular}

Su representación actancial nos mostrará la dinámica establecida entre los diferentes planos de dicha estructura paralela y será la siguiente:

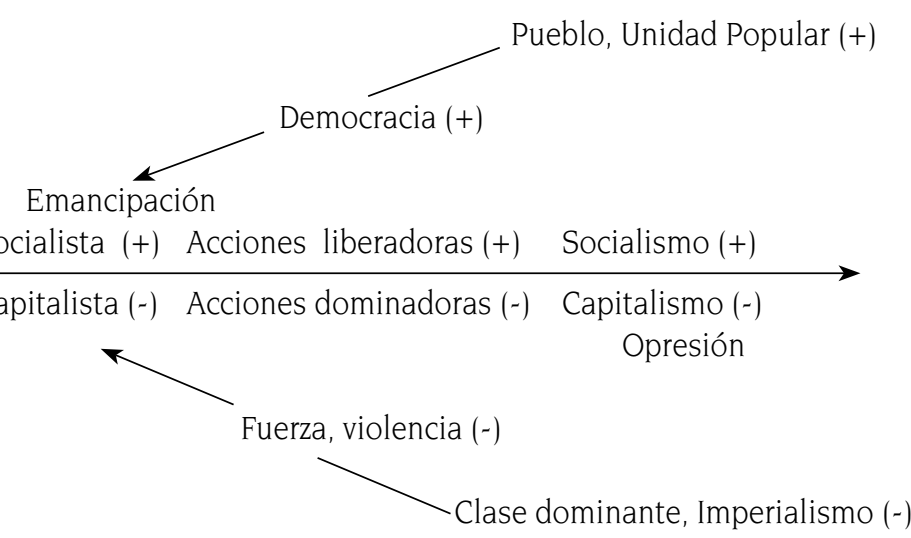

De un lado, en la polaridad negativa, vemos que el hombre capitalista ejerce acciones de dominación, a través de la fuerza y la violencia y que son legitimadas por la clase dominante y el imperialismo con el fin de oprimir a otros y así producir y reproducir el capitalismo. Del otro lado, en la polaridad positiva, el ser socialista se provee de acciones liberadoras a través de la democracia y que legitiman y son legitimadas por el pueblo -representado en la Unidad Popular-buscando como finalidad construir la sociedad socialista.

Un análisis en profundidad de lo expresado en la estructura nos permitirá mostrar las potencialidades analíticas del MAE y, concretamente, de esta estructura. Veamos esta dinámica en su proceso histórico concreto.

Debemos señalar que la estructura actancial encontrada es fundamentalmente de carácter sociopolítico y responde -de alguna manera- a un período histórico de la humanidad, en que el mundo quedó dividido en dos polos, a consecuencia de la "guerra fría", así 
como también, a la situación particular que vivía Chile y los pueblos latinoamericanos en la década de los años 60.

En efecto, ya desde fines de los años cincuenta, en Chile, los límites de las políticas económicas iniciadas bajo los gobiernos anteriores se habían hecho evidentes. Se había logrado avanzar en un importante proceso de industrialización en el país, pero la industria era débil y dependiente, el mercado nacional pequeño y los recursos estatales reducidos: dependían principalmente del cobre, que permanecía en manos norteamericanas. No se habían nacionalizado las riquezas básicas ni tampoco se había realizado una reforma agraria que dinamizara la producción del agro, manteniéndose en consecuencia las viejas relaciones de producción y propiedad basadas en el latifundio. No se habían tocado tampoco los intereses de la vieja oligarquía, que se había venido fusionando con la nueva clase empresarial.

Los mayores beneficios de la etapa de expansión industrial y de servicios habían favorecido más a los sectores dominantes y a la clase media que a los sectores populares. En los años sesenta, Chile era un país capitalista dependiente que no lograba remontar una crisis de crecimiento económico, que se expresaba principalmente en estancamiento, inflación, escasez de divisas y agudización de los problemas sociales y de subsistencia de las mayorías populares.

Como consecuencia de lo anterior, una creciente mayoría nacional se mostraba partidaria de modificar la estructura económica, social y política del país. Las propuestas de cambio que animan a la sociedad en esos años encuentran sus antecedentes también en el progresivo proceso de democratización que había venido viviendo la sociedad chilena desde los años cuarenta.

Por otro lado, la realización del Concilio Vaticano II, en el que se decide dar mayor atención a los problemas sociales que vive el mundo, va a provocar en la Iglesia chilena un paulatino alejamiento de los conservadores y un progresivo acercamiento al mundo de los pobres.

Asimismo, la ola de movimientos populares que recorría a América Latina en esos años, luego del triunfo de la revolución Cubana, contribuyeron a afianzar la voluntad política de cambios en el país. 
El discurso analizado responde, por tanto, a condiciones históricas, socio-políticas y económicas particulares de Chile en dicha década y encarna la esperanza y voluntad de cambio de vastos sectores de la sociedad chilena, lo que en cierta medida "justifica" el porqué del discurso dicotómico capitalismo/socialismo.

\section{Referencias bibliográficas}

HIERNAUX, J. P. (1977). L'institution culturelle. Méthode de description structurale. 1977. Louvain-la-Neuve: Presses Universitaires de Louvain.

HIERNAUX, J. P. (1994). Et hic tres unum sunt. Structures croisées et théorie des réductions. Notes de travail.

HIERNAUX, J. P. (1995). Analyse structurale de contenus et modèles culturels. Comment traiter des matériaux volumineux. In Albarello y Digneffe Pratiques et methodes de recherche en Sciences Sociales. París: Armand Colin.

PIRET, A., NIZET, J. Y BOURGEOIS, E. (1996). L'analyse structurale, Une méthode d'analyse de contenu pour les sciences humaines. Paris: De Boeck Université. 
